Bangl. J. Vet. Med. (2007). 5 (1 \& 2): 39-42

\title{
SEROPREVALENCE OF SALMONELLOSIS IN LAYER CHICKENS WITH ISOLATION, IDENTIFICATION AND ANTIBIOGRAM STUDY OF THEIR CAUSAL AGENTS
}

\author{
M. R. Akter ${ }^{1}$, K. A. Choudhury, M. M. Rahman ${ }^{2}$ and M. S. Islam ${ }^{1}$ \\ Department of Microbiology and Hygiene, Faculty of Veterinary Science, Bangladesh Agricultural University, \\ Mymensingh-2202, Bangladesh

\begin{abstract}
Salmonellosis is a common problem in poultry farms of our country. Indiscriminate use of antibiotic to control the disease results drug resistance and limits the therapeutic possibilities in the treatment of the disease. This study was conducted during the period from January to May 2006 at Gobindapur of Dinajpur district. The present study was undertaken to determine the seroprevalence of salmonellosis in layer flocks and antibiogram study following isolation of Salmonellae. A total of $225 \mathrm{Star}$ cross 579 brown chickens were studied with rapid serum plate agglutination test. Liver of 200 dead birds was studied for isolation and identification of Salmonellae. In vitro antibiotic sensitivity test of isolated Salmonellae was performed with commercial sensitivity discs. The overall seroprevalence was recorded $23.11 \%$. The prevalence was varied from age to age. The highest rate was $28 \%$ in above 20 weeks of age. The antibiogram study revealed that the isolates were sensitive to ciprofloxacin $(80 \%)$, nitrofurantoin $(100 \%)$, sulphamethoxazole/ trimeoprim and amoxycillin $(50 \%)$, tetracycline $(60 \%)$ but resistant to penicillin-G and erythromycin. Further studies should be conducted on serotyping of the isolated Salmonellae, isolation and identification of Salmonellae from different feed and environmental sample.
\end{abstract}

Key words: Salmonellosis, seroprevalence, antibiogram, layer chickens

\section{INTRODUCTION}

Salmonellosis in poultry causes heavy economic loss through mortality and reduced production (Khan et al., 1998). The disease is most significant because the causal agents of the disease are transmitted vertically from parent to offspring. Vaccines form local isolates commercially still not available in the market for effective preventive measure. So, the control of the disease mainly relies on the use of antimicrobial drugs. This leads to indiscriminate use of antimicrobial drugs in poultry industry that results antibiotic resistance and limits the therapeutic possibilities in the treatment of bacterial diseases. In Bangladesh, the Salmonella infections in chicks and layer chickens must be evaluated for effective control measures of the diseases (Islam et al., 2006). Considering these facts, a study was undertaken to determine the sero-prevalence and clinical-prevalence of salmonellosis in layer flock as well as antibiotic sensitivity test following isolation of Salmonellae from those flocks.

\section{MATERIALS AND METHODS}

This study was conducted during the period from January to May 2006 at Gobindapur of Dinajpur district in 'Star Agro Poultry Farm'. The samples were collected from the birds of selected layer farm and brought to the Department of Microbiology, Dinajpur Government Veterinary College for laboratory analysis. The breed was Star cross 579 brown. The birds and their parents were not vaccinated with any Salmonella vaccine. No antibiotics were used previously in the selected flocks for the prevention of any disease. The birds were divided into three groups as group A ( $~ 8$ weeks old), group B (9-20 weeks old) and group C (above 20 weeks old).

\footnotetext{
${ }^{1}$ Department of Physiology, Biochemistry and Pharmacology, ${ }^{2}$ Department Microbiology, Dinajpur Government Veterinary College, Basherhat, Dinajpur.
} 


\section{Seroprevalence study}

\section{Sample collection}

A total of 225 blood samples were collected from selected flocks (10\% of total flock) from the wing vein of individual birds. Blood were collected aseptically in sterile vial with sterile 5-ml syringe. Then the samples were allowed to clotting in the syringe and kept for 1-2 hours at room temperature, after clotting, sera were separated, centrifuged and poured in sterile vials, labeled individually and stored at $-20^{\circ} \mathrm{C}$ until used.

\section{Rapid serum plate agglutination test}

The rapid serum plate agglutination test was preformed according to the procedure described by OIE (2000) with crystal violet stained Salmonella antigen (Nobilis ${ }^{(\mathrm{R})} \mathrm{SP}$ antigen). For this test $0.02 \mathrm{ml}$ of antigen and 0.02 $\mathrm{ml}$ of chicken sera were placed side by side with a micropipette on a glass plate and was mixed thoroughly by stirring with stirrer stick followed by rocking. The results were observed within 2 minutes. In positive cases granules were formed slowly which was seen during rocking. In the absence of antibody, no such granules were formed within two minutes. All rapid serum plate agglutination test results were recorded.

\section{Isolation and identification of causal agent}

\section{Sample collection}

Liver of a total of 200 dead birds from different flock (Group A- 70, Group B-60 and Group C-70) were collected and brought to the Department of Microbiology, Dinajpur Government Veterinary College, Dinajpur. Isolation and identification of Salmonellae were performed as per procedure described by OIE (2000), Merchant and Packer (1967) and Cowan (1985). Salmonellae sample were isolated from the collected liver samples by sterilized inoculation loop. Primary culture was performed in nutrient agar. Subcultures were performed in blood agar, MacConkey (MC) agar and Salmonella-Shigella (SS) agar to get pure culture and cultural characteristics.

\section{Morphological characterization}

The representative Salmonellae isolates from SS agar were stained by Gram's stain (Merchant and Packer, 1967). Motility test was performed by MIU (Motility, Indole, Urea) medium according to the procedure described by OIE (2000).

\section{Biochemical test}

Several biochemical tests such citrate utilization test (using Simmons citrate agar), triple sugar iron (TSI) agar slant reaction, Indole test, Methyl Red (MR) test, Voges-Proskaur (V-P) test, Dulcitol fermentation test and Ornithine test were performed according to the procedure described by Cowan (1967), Merchant and Packer (1967) and OIE (2000).

\section{Antibiotic sensitivity test}

In vitro antibiotic sensitivity test of isolated Salmonella was performed with the standardized commercial sensitivity discs manufactured by Oxoid limited (Basingstoke, Hampshire, England). Sensitivity to antibacterial agents (antibiotic) was studied on nutrient agar and Salmonella-Shigella agar plates with erythromycin (E) $15 \mu \mathrm{g}$, sulphamethoxazole/ trimethoprim (SxT) $25 \mu \mathrm{g}$, ciprofloxacin (CIP) $5 \mu \mathrm{g}$, nitrofurantoin (F) 300 $\mu \mathrm{g}$, amoxycillin (AML) $25 \mu \mathrm{g}$, penecilin-G (P) 10 units and tetracycline (TE) $30 \mu \mathrm{g}$. This test was performed according to the procedure described in OIE (2000).

\section{RESULTS AND DISCUSSION}

\section{Seroprevalence study}

The overall prevalence of salmonellosis was detected as $23.11 \%$ (Table 1). This finding is supported by Alam et al. (2003) and Sikder et al. (2005) who observed 23.8\% and 23.46\% prevalence, respectively. But Torzolo et al. (1977), Prukner (1987), Ghosh (1988), Waltman and Horne (1993) and Yang et al. (1996) reported 9\%, $13.9 \%, 7.5 \%, 10 \%$ and $15 \%$ prevalence of salmonellosis respectively which may be due to geographical variation. The prevalence was varied in terms of age (Table 1). It indicated that seroprevalence varied with the increase of age of the birds, which supports the findings of Truong and Tieuquang (2003), Sikder et al. (2005) and Islam et al. (2006). 
Salmonellosis in layer chickens

Table 1. Seroprevalence of salmonellosis in layer birds

\begin{tabular}{|lllll|}
\hline Groups & Age (weeks) & No. of sera sample tested & Positive case & Seroprevalence (\%) \\
\hline A & $\sim 8$ & 100 & 20 & 20.00 \\
B & $9-20$ & 75 & 18 & 24.00 \\
C & $>20$ & 50 & 14 & 28.00 \\
\hline Total & 225 & 52 & 23.11 \\
\hline
\end{tabular}

\section{Isolation of causal agent}

A total of $15(7.5 \%)$ Salmonellae were isolated from 200 liver of dead birds of selected flocks of different groups and characterized by using specific biochemical tests and Gram's staining technique (Table 2). The rate of isolation was slightly lower than Hossain et al. (2006) but markedly lower than Islam et al. (2006). This may be due to variation of severity. It was observed that the highest rate $(12.98 \%)$ of Salmonella was isolated from group C followed by group A $(5.71 \%)$ and group B (3.33\%). This finding is supported by Islam et al. (2006), Lee et al. (2001) and Hoque et al. (1996).

Table 2. Isolation rate of Salmonella from different groups of birds

\begin{tabular}{|llll|}
\hline Group of birds & No. of liver sample studied & No. of positive cases & Isolation rate (\%) \\
\hline A & 70 & 4 & 5.71 \\
B & 60 & 2 & 3.33 \\
C & 70 & 9 & 12.86 \\
\hline Total & 200 & 15 & 7.50 \\
\hline
\end{tabular}

Group A = $>8$ weeks, Group B = 9-20 weeks, Group A = >20 weeks

It was observed that all isolates were gram negative, small rod shaped with single or pair shaped. All the isolates were indole negative and TSI agar slant positive. Out of 15 isolates 12 were positive on dulcitol and others were negative. On the other hand 3 were ornithine positive and other 12 were negative. It indicated that the $12(80 \%)$ isolates were Salmonella gallinarum and $3(20 \%)$ were Salmonella pullorum. The higher rate of Salmonella gallinarum than S. pullorum is supported by Hossain et al. (2006) who detected $62.5 \%$ S. gallinarum and $25 \%$ S. pullorum.

\section{Antibiotic sensitivity of Salmonella isolates}

The antibiogram study revealed that the isolates were sensitive to ciprofloxacin $(80 \%)$, nitrofurantoin $(100 \%)$, sulphamethoxazole/trimethoprim and amoxycillin (50\%), tetracycline (60\%) (Table 3$)$. The isolates were found resistant to penicillin-G and erythromycin but nitrofurantoin was found highly effective $(100 \%)$. These findings were strongly supported by Verma et al. (1993), Anjanappa et al. (1994) and Hui and Das (2001).

Table 3. Antibiotic sensitivity of the isolated Salmonellae

\begin{tabular}{|l|llll|}
\hline Antibacterial agents & Sensitive* & \multicolumn{3}{l|}{ Resistance } \\
\cline { 2 - 5 } & No. & $\%$ & No. & $\%$ \\
\hline Ciprofloxacin & 8 & 2 & 80 & 20 \\
Nitrofurantoin & 10 & 0 & 100 & 00 \\
Tetracycline & 6 & 4 & 60 & 40 \\
Sulphamethoxazole/Trimethoprim & 5 & 5 & 50 & 50 \\
Erythromycin & 0 & 10 & 00 & 100 \\
Penicillin-G & 0 & 10 & 00 & 100 \\
Amoxycillin & 5 & 5 & 50 & 50 \\
\hline
\end{tabular}

$* \geq 2 \mathrm{~mm}$ clear zone around the antibiotic disc indicated sensitive cases.

However, for useful application of the present research findings further studies should be conducted on serotyping of the isolated Salmonellae, isolation and identification of Salmonellae from different feed and environmental sample. 


\section{REFERENCES}

1. Alam J, Koike I, Giasuddin M and Rahman M (2003). Seroprevalence of poultry diseases in native chickens in Bangladesh. $9^{\text {th }}$ BSVER Annual Scientific Conference, BSVER Publication No. 24 . p. 26.

2. Anjanappa M, Harbola PC and Verma JC (1994). Plasmid profile analysis of field strain of Salmonella gallinarum. Indian Journal of Comparative Microbiology, Immunology and Infectious Diseases 16: 79.

3. Cowan ST (1985). Cowan and Steel's Manual for the Identification of Medical Bacteria. $2^{\text {nd }}$ edn., New Age International (P) Ltd., New Delhi, India.

4. Ghosh SS (1988). Incidence of pullorum disease in Nagaland. Indian Veterinary Journal 62 (10): 949-951.

5. Hoque MM, Biswas HR and Rahman L (1996). Isolation, identification and production of Salmonella pullorum coloured antigen in Bangladesh for the rapid whole blood test. Asian-Australasian Journal of Animal Sciences 10 (1): 141-146.

6. Hossain MS, Chowdhury EH, Islam MM, Haider MG and Hossain MM (2006). Avian Salmonella infection: Isolation and identification of organisms and histopathological study. Bangladesh Journal of Veterinary Medicine 4 (1): 07-12.

7. Hui AK and Das R (2001). Studies on isolation, serotyping and antibiotic sensitivity of Salmonellae isolated from ducks. Indian Veterinary Journal 78 (11): 1058-1059.

8. Islam MM, Haider MG, Chowdhury EH, Kamruzzaman M and Hossain MM (2006). Seroprevalence and pathological study of Salmonella infections in layer chickens and isolation and identification of causal agents. Bangladesh Journal of Veterinary Medicine 4 (2): 79-85.

9. Khan MAHNA, Bari ASM, Islam MR, Das PM and Ali MY (1998). Pullorum disease in seminature chicks and its experimental pathology. Bangladesh Veterinary Journal 32: 124-128.

10. Lee YJ, Kang MS, Woo YK, Mo IP and Tak RB (2001). Competitive exclusion against Salmonella gallinarum of Salmonella enteritidis infected chickens. Journal of Veterinary Science 2: 33-36.

11. Merchant IA and Packer RA (1967). Veterinary Bacteriology and Virology. $7^{\text {th }}$ edn., The Iowa State University Press, Ames, Iowa, USA.

12. OIE (Office International Des Epizooties) (2000). Manual of standards for diagnostics test and vaccines. OIE Guide-2.

13. Prukner E (1987). Bacterial infections in the etiology of poultry diseases. Peradarstvo 22 (11-12): 295-298.

14. Sikder AJ, Islam MA, Rahman MM and Rahman MB (2005). Seroprevalence of Salmonella and Mycoplasma gallisepticum infection in the six model farms at Patuakhali district of Bangladesh. International Journal of Poultry Science 4: 905-910.

15. Torzolo HR, Verona AZde, D'Furowicz AJJ and De-Verona AZ (1977). Finding in fowls Escherichia coli strains having common antigens with Salmonella genus. Revista-de-la-Asociacion-Agrentina-de-Microbiologia 9 (1): 4-10.

16. Truong Q and Tieuquang AN (2003). Prevalence of Salmonella gallinarum and pullorum infection in the luong Phuong chickens reared in the household sector. Khoa-Hoc-Ky-Thuat-Thu-Y-Veterinary-Science-and-Techniques 10: 15-19.

17. Verma JG, Gupta BR and Ghosh SS (1993). Studies on Salmonella virchow: in vitro sensitivity. Indian Veterinary Journal 70: 572-573.

18. Waltman WD and Horne AM (1993). Isolation of Salmonella from chickens reacting in the pullorum typhoid agglutination test. Avian Diseases 37 (3): 805-810.

19. Yang YC, Sun Y, Wang MM, Li YK, Zhang XX and Sun BA (1996). Discussion of on site detection of pullorum disease in breeding chickens and control measures. Chinese Journal of Veterinary Medicine 22 (7): 20-22. 